\title{
Impedance to retrograde and forward flow in chronic mitral regurgitation and the physiology of a double outlet ventricle
}

\author{
William H Gaasch, ${ }^{1}$ Sachin P Shah, ${ }^{1}$ Sherif B Labib, ${ }^{1}$ Theo E Meyer ${ }^{2}$
}

'Department of Cardiovascular Medicine, Lahey Hospital and Medical Center, Burlington, Massachusetts, USA 2Department of Cardiovascular Medicine, University of Massachusetts Medical Center, Worcester, Massachusetts, USA

\section{Correspondence to} William H Gaasch, Department of Cardiovascular Medicine, Lahey Hospital and Medical Center, 41 Mall Road, Burlington MA, 01805, USA william.h.gaasch@lahey.org

Received 3 April 2016 Revised 2 September 2016 Accepted 3 September 2016 Published Online First 28 September 2016

\section{Linked}

- http://dx.doi.org/10.1136/ heartinl-2016-310472

- http://dx.doi.org/10.1136/ heartjnl-2016-311009

CrossMark

To cite: Gaasch WH

Shah SP, Labib SB, et al. Heart 2017:103:581-585.

\section{ABSTRACT}

Objective Mitral regurgitation (MR) is generally characterised as exhibiting a 'low impedance leak into the left atrium'. This notion is widely accepted without measured impedance data. The aim of this study was to define the impedance to retrograde and forward blood flow and to examine hydraulic (pressure-volume) and mechanical (stress-shortening) function in chronic severe MR.

Methods A mathematical model of a double outlet ventricle was developed and the ratio of retrograde to forward impedance was plotted over a wide range of regurgitant fraction (RF). The model predicts that an impedance ratio $>1$ indicates that the impedance to retrograde flow exceeds that of forward flow. Left ventricular (LV) systolic pressure/flow rate was used as an index of impedance $(\mathrm{mm} \mathrm{Hg} / \mathrm{mL} / \mathrm{s})$. Data from 10 patients with severe MR were used to assess the clinical applicability of the model. All patients had degenerative valve disease with partial flail leaflet, an $\mathrm{RF}>50 \%$ and an ejection fraction $(E F)>0.60$. There were seven males and three females, aged $59 \pm 10$. LV volumes as well as retrograde and forward flow rates were determined with echocardiographic and Doppler techniques.

Results The model indicates that the impedance ratio is $>1$ when the RF ranges from zero to $57 \%$. Clinical data: end-diastolic volume $=184 \pm 47 \mathrm{~mL} ; E F=0.63 \pm 3 \%$; $\mathrm{RF}=53 \pm 4 \%$. Values for retrograde and forward impedance were $0.77 \pm 0.17$ and $0.63 \pm 0.12(p=0.003)$; the impedance ratio was $1.22 \pm 0.19$. Total impedance to LV emptying was low $(0.35 \pm 0.06)$. The ratio of systolic wall stress to EF $\left(580 \pm 81 \mathrm{~g} / \mathrm{cm}^{2}\right)$ was normal. Data are mean \pm SD.

Conclusions The model, supported by clinical data, indicates that the impedance to retrograde flow exceeds the impedance to forward flow in chronic severe MR. These findings refute the notion of a low impedance leak into the left atrium. The double outlet of an enlarged ventricle provides a mechanism for low total impedance to ejection in the presence of a normal stress-shortening relation.

\section{INTRODUCTION}

Chronic mitral regurgitation (MR) is described as a condition exhibiting a 'low impedance leak into the left atrium' that facilitates ventricular emptying. ${ }^{1}$ Many authorities subscribe to this haemodynamic model, but the concept of impedance as an opposition or resistance to blood flow has often been used without any measurements of impedance. Despite a lack of published impedance data in patients with MR, the concept of a low impedance outlet into the left atrium is generally unquestioned and the notion of a low impedance 'unloading' effect is widely accepted. ${ }^{2}$ However, these concepts are not in accordance with published data indicating that left ventricular(LV) afterload is not low in chronic MR. Indeed, afterload remains in the normal range in compensated $\mathrm{MR}$ and tends to increase during the development of ventricular dysfunction. ${ }^{3}$ This apparent discrepancy between the notion of a low impedance unloading effect and published data indicating normal or increased afterload has not been reconciled in chronic severe MR.

The primary goal of the study was to scrutinise and assess the concept of a low impedance pathway into the left atrium in chronic MR. This valve lesion was modelled as a double outlet ventricle and the impedance to retrograde blood flow and its relation to forward blood flow was determined in chronic severe MR. To evaluate what appears to be a discrepancy between the loading effects of impedance and afterload, the total impedance to ejection and the $\mathrm{LV}$ afterload-shortening relation were examined. In this study, the term impedance is used to indicate a hydraulic opposition or obstruction to blood flow. Such a hydraulic load differs from afterload which is a myocardial mechanics term that reflects the LV wall forces resisting myocardial shortening. ${ }^{4}$

\section{METHODS}

First, a mathematical model of a double outlet ventricle was developed in order to illustrate the relative magnitude of retrograde and forward impedance over a wide range of regurgitant fractions. Then, clinical data from 10 patients with MR were used to evaluate the applicability of the model. Finally, the impedance results were analysed in concert with systolic wall stress data and an attempt was made to reconcile any nominal disparity between the concepts of these hydraulic and mechanical loads.

\section{The double outlet model}

A relatively simple mathematical model was developed. The model incorporates an end-diastolic volume (EDV) of $180 \mathrm{~mL}$, an ejection fraction (EF) of $60 \%$ and a LV mean systolic pressure (MSP) of $110 \mathrm{~mm} \mathrm{Hg}$. These values are typical of what is seen in chronic compensated severe MR. ${ }^{2}{ }^{3}$ Using this information, total stroke volume (SV) was calculated as the product of EDV and EF. The mitral regurgitant volume (MRV) and the forward SV, 
determined over a wide range of regurgitant fractions (20\%$80 \%)$, were calculated as:

$$
\begin{aligned}
& \mathrm{MRV}=\text { total } \mathrm{SV} \times \text { regurgitant fraction } \\
& \text { Forward } \mathrm{SV}=\text { total } \mathrm{SV}-\mathrm{MRV}
\end{aligned}
$$

The model incorporates an index of impedance that was modified from the method by Briand et al. ${ }^{6}$ This method which uses the ratio of LV systolic pressure to $\mathrm{SV}$ is thought to represent LV outflow impedance (aka valvuloarterial impedance); it appears to have prognostic value in patients with aortic stenosis. ${ }^{6}$ In the absence of heart valve disease, this measure of impedance is closely related to effective arterial elastance (aka arterial load). ${ }^{8}$ Such a ratio is appropriate in a single outlet ventricle, but in the double outlet ventricle of MR consideration should be given to the fact that the duration of retrograde flow exceeds that of forward flow. Therefore, it is necessary for the model to incorporate different durations of retrograde and forward flow; in the model, 400 and 300 ms were used. ${ }^{9}$ Thus, retrograde and forward flow rates were calculated as MRV/400 ms and forward SV/300 ms. The index of impedance used in the model was calculated as MSP divided by flow rate and expressed in $\mathrm{mm} \mathrm{Hg} / \mathrm{mL} / \mathrm{s}$.

$$
\begin{aligned}
& \text { Retrograde impedance }=110 \div \mathrm{MRV} / 400 \mathrm{~ms} \\
& \text { Forward impedance }=110 \div \text { forward SV } / 300 \mathrm{~ms}
\end{aligned}
$$

In figure 1 (upper panel), retrograde and forward impedance values are shown at $10 \%$ intervals over a range of regurgitant fractions. In the lower panel, the ratio of retrograde to forward impedance is plotted against the regurgitant fraction. The model illustrates that a ratio exceeding 1 indicates that the impedance to retrograde flow exceeds that of forward flow.

\section{Clinical application}

Using the echocardiography laboratory database, patients with severe MR were identified using the American Society of Echocardiography 'integrative approach' that relies on multiple echocardiographic parameters. ${ }^{10}$ Ten patients with chronic severe MR and high-quality echocardiograms and Doppler studies were selected. All 10 patients had degenerative mitral valve disease with partial flail leaflet, holosystolic MR, LV enlargement, a normal EF and a regurgitant fraction exceeding 50\%. There were seven males and three females, with ages ranging from 43 to 77 years $(59 \pm 10)$. All patients had mild symptoms of dyspnoea and fatigue (functional class II). None was being treated with antihypertensive agents or vasodilators. None had coronary artery disease or regional wall motion abnormalities and none was considered to have secondary or functional MR. This clinical portion of the study consisted of a retrospective review and analysis of echocardiographic records and was approved by the Institutional Review Board at the Lahey Hospital and Medical Center.

Two-dimensional and Doppler echocardiograms were obtained using the same standard techniques that were used in our previous studies. ${ }^{11} 12$ The EDV, total SV and EF were calculated with the angiography-based echocardiographic method by Teichholz et al. ${ }^{13}$ The forward SV was calculated as the product
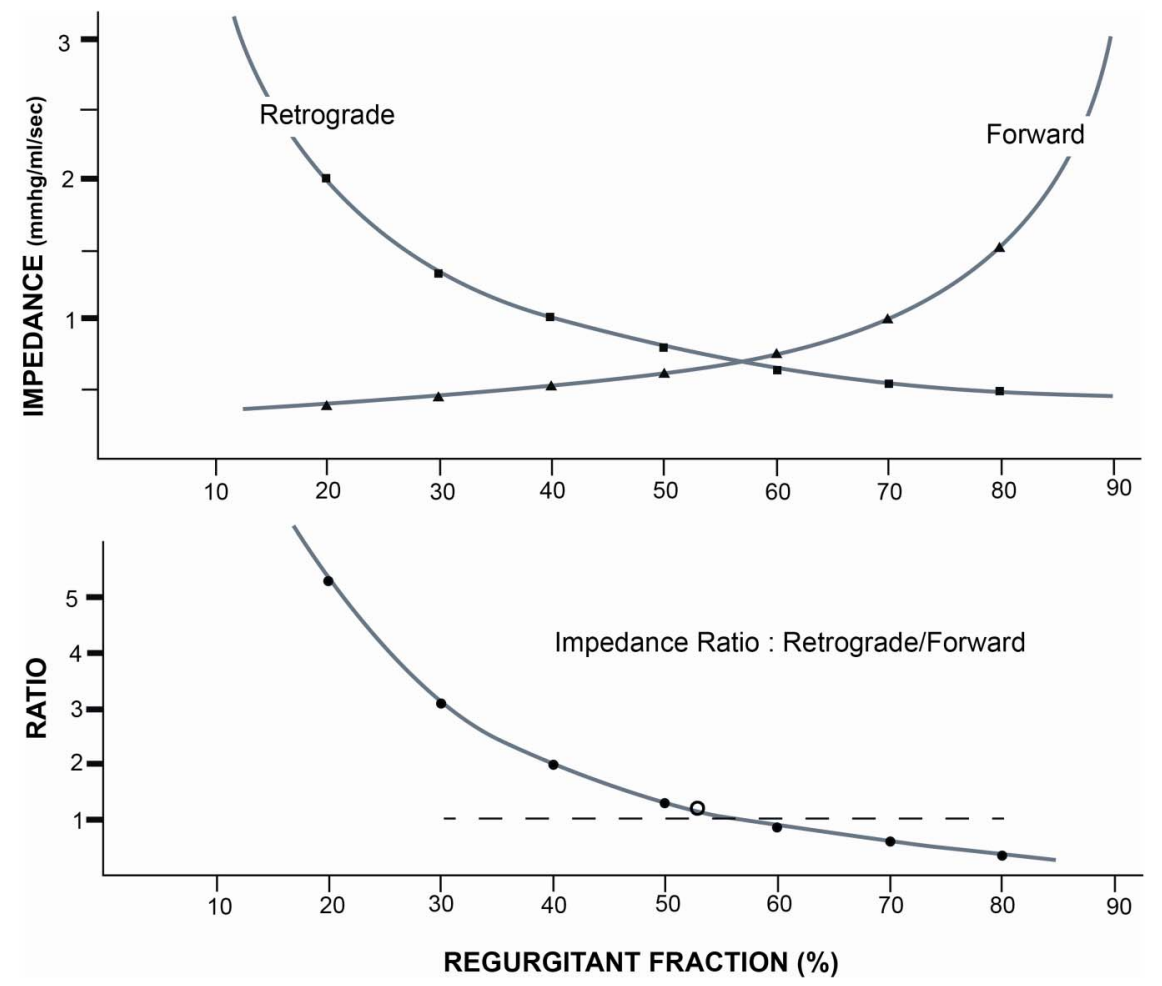

Figure 1 Impedance to retrograde and forward flow in chronic mitral regurgitation. The model is based on an end-diastolic volume of $180 \mathrm{~mL}$, an ejection fraction of $60 \%$ and a left ventricular mean systolic pressure of $110 \mathrm{~mm} \mathrm{Hg}$. The durations of retrograde and forward flow were taken as 400 and $300 \mathrm{~ms}$, respectively. In the upper panel, the impedance to retrograde flow (closed squares) is plotted against regurgitant fraction; the coordinates were calculated over a wide range of regurgitant fractions (at intervals of 10\%). Likewise, the impedance to forward flow (closed triangles) is plotted against regurgitant flow over the same range of regurgitant fractions. The impedance to retrograde flow is greater than forward flow over a wide range of regurgitant fractions up to $57 \%$. Only when the regurgitant fraction exceeds $57 \%$ is the impedance to retrograde flow less than to forward flow. In the lower panel, the ratio of retrograde to forward impedance (closed circles) is plotted against regurgitant fraction. The model indicates that a ratio exceeding one (broken line) reflects a higher impedance to retrograde flow than to forward flow. The average ratio $(1.22 \pm 0.19)$ of the patient group with a regurgitant fraction of $53 \pm 4 \%$ (open circle) is superimposed on the model. 
of the LV outflow tract cross-sectional area and the outflow tract time-velocity integral. ${ }^{14}$ The MRV was determined by subtracting the forward SV from the total SV.

$$
\mathrm{MRV}=\text { total SV }- \text { forward SV }
$$

LV mass was calculated using the necropsy-based echocardiographic method by Devereux et al. ${ }^{15}$ The dimensionless mass/ volume ratio was derived as the ratio of $\mathrm{LV}$ wall volume to EDV, where wall volume was derived as mass divided by $1.05 .^{16}$ These volume and mass data were applied in the calculations of impedance and wall stress.

Impedance was calculated as described for the model (vide supra), but here the calculations included measured durations of retrograde and forward flow. In each patient, the duration of retrograde flow and forward flow was measured from the continuous wave Doppler signals of the regurgitant and forward flows. Thus, retrograde flow rate was calculated as retrograde flow divided by the duration of regurgitation. In a similar fashion, forward flow rate was calculated as forward flow divided by forward ejection time. The index of impedance was calculated as MSP $(\mathrm{mm} \mathrm{Hg})$ divided by flow rate $(\mathrm{mL} / \mathrm{s})$. In this calculation and in those to follow, MSP and end-systolic pressure were derived as the product of systolic blood pressure (sphygmomanometer) and $0.9 .{ }^{8}$ Total impedance $\left(\mathrm{I}_{\mathrm{t}}\right)$ to $\mathrm{LV}$ ejection in the double outlet ventricle was calculated as impedance in a parallel circuit. ${ }^{17}$ Thus, the reciprocal of total impedance equals the sum of the reciprocals of the individual values:

$$
1 / \mathrm{I}_{\mathrm{t}}=1 / \mathrm{I}_{\mathrm{f}}+1 / \mathrm{I}_{\mathrm{r}},
$$

where $I_{f}=$ forward impedance and $I_{r}=$ retrograde impedance. This parameter $\left(\mathrm{I}_{\mathrm{t}}\right)$ reflects the sum effect of the two outlets and it provides a measure of the total hydraulic opposition to $\mathrm{LV}$ emptying.

The resistances to forward and retrograde blood flow were determined using standard formulae. ${ }^{18}$ The resistance of the mitral regurgitant orifice was calculated as the transmitral systolic pressure gradient divided by the retrograde flow rate. The pressure gradient was approximated as the LV MSP minus left atrial pressure; the latter was assumed to be $20 \mathrm{~mm} \mathrm{Hg}$. The resistance to forward flow was taken as the systemic vascular resistance. Resistance per beat is expressed in dyne $\mathrm{s} / \mathrm{cm}^{5}$.

Afterload was defined as systolic wall stress, which is calculated as a force per unit cross-sectional area of the LV wall. Stress (S) was calculated as: $\mathrm{S}=\mathrm{P} \times(1+3 \mathrm{~V} / \mathrm{M})$, where $\mathrm{P}=\mathrm{LV}$ systolic pressure, $\mathrm{V}=\mathrm{LV}$ chamber volume and $\mathrm{M}=\mathrm{LV}$ wall volume. ${ }^{19}$ Recognising that wall stress varies throughout systole, we calculated this parameter at the moment of aortic valve opening, at the time of peak stress and at the end of systole. ${ }^{71120}$ Mean systolic wall stress was determined as the average of these three stress values. The mean stress values reported herein represent the mechanical load that opposes myocardial shortening. Stress values are given in $\mathrm{g} / \mathrm{cm}^{2}$.

\section{Analysis}

Student's t-test for paired data was applied to assess differences between retrograde and forward impedance and resistance values, as well as for retrograde and forward impedance versus total impedance. Data are presented as mean \pm SD. p Values $<0.05$ were considered to be statistically significant.

\section{RESULTS}

Haemodynamic data from the 10 patients are shown in table 1. All patients exhibited LV enlargement with eccentric
Table 1 Echocardiographic data in chronic severe mitral regurgitation

\begin{tabular}{lc}
\hline Body surface area $\left(\mathrm{m}^{2}\right)$ & $1.9 \pm 0.1$ \\
Systolic blood pressure $(\mathrm{mm} \mathrm{Hg})$ & $126 \pm 10$ \\
Diastolic blood pressure $(\mathrm{mm} \mathrm{Hg})$ & $75 \pm 9$ \\
LV end-diastolic dimension $(\mathrm{mm})$ & $61 \pm 6$ \\
LV wall mass $(\mathrm{g})$ & $217 \pm 75$ \\
LV end-diastolic volume $(\mathrm{mL})$ & $183 \pm 47$ \\
Mass/volume & $1.1 \pm 0.1$ \\
Total stroke volume $(\mathrm{mL})$ & $117 \pm 27$ \\
Ejection fraction $(\%)$ & $64 \pm 3$ \\
Forward stroke volume $(\mathrm{mL})$ & $54 \pm 10$ \\
Regurgitant volume $(\mathrm{mL})$ & $63 \pm 20$ \\
Regurgitant fraction $(\%)$ & $53 \pm 4$ \\
\hline LV left ventricular. &
\end{tabular}

hypertrophy, a normal mass/volume ratio, an EF exceeding 60\% and a regurgitant fraction exceeding 50\%. The duration of forward flow was $294 \pm 22 \mathrm{~ms}$. The duration of regurgitant flow was $413 \pm 17 \mathrm{~ms}$. These data are consistent with the diagnosis of chronic severe MR.

\section{Impedance}

The model (shown in figure 1) indicates that impedance to retrograde flow is substantially higher than the impedance to forward flow in mild and moderate MR (ie, regurgitant fraction $<50 \%$ ). At a regurgitant fraction of $57 \%$, the ratio of retrograde to forward impedance is one, indicating that the impedance to retrograde flow is equal to that of forward flow. At higher regurgitant fractions (ie, $>57 \%$ ), the ratio falls below one, indicating that the impedance to retrograde flow is less than that to forward flow.

In the patients with MR, the average values for impedance and resistance to regurgitant flow were significantly higher than those to forward flow (table 2); the average regurgitant fraction was $53 \%$ and the ratio of retrograde to forward impedance was $1.22 \pm 0.19$. This value, shown in the figure as an open circle, is in close agreement with the model. The total impedance to ejection was subnormal (range: 0.22-0.44, mean \pm SD: 0.35 $\pm 0.06 \mathrm{~mm} \mathrm{Hg} / \mathrm{mL} / \mathrm{s}$ ), and significantly less than the impedance to retrograde flow (range: $0.40-1.01$, mean \pm SD: $0.77 \pm 0.17$, $\mathrm{p}=0.0001$ ) as well as that to forward flow (range: $0.48-0.86$, mean \pm SD: $0.63 \pm 0.12, p=0.0001)$ ). This total impedance result is what might be expected in an enlarged double outlet ventricle with a normal EF. These observations support the concept of a reduced total hydraulic load in chronic MR, but they are not in accordance with the notion of a 'low impedance leak' in all patients with MR.

Table 2 Impedance, resistance and systolic wall stress data in chronic severe mitral regurgitation

\begin{tabular}{ll}
\hline Impedance $(\mathrm{mm} \mathrm{Hg} / \mathrm{mL} / \mathrm{s})$ & \\
$\quad$ Retrograde & $0.77 \pm 0.17$ \\
$\quad$ Forward & $0.63 \pm 0.12, \mathrm{p}=0.003$ vs retrograde \\
$\quad$ Total & $0.35 \pm 0.06$ \\
Resistance $\left(\right.$ dyne $\left.\mathrm{s} / \mathrm{cm}^{5}\right)$ & $851 \pm 191$ \\
$\quad$ Retrograde & $674 \pm 130, \mathrm{p}=0.001$ vs retrograde \\
$\quad$ Forward & \\
Afterload $\left(\mathrm{g} / \mathrm{cm}^{2}\right)$ & $372 \pm 24$ \\
$\quad$ Mean systolic stress & \\
\hline
\end{tabular}




\section{Afterload}

Systolic wall stress data are shown in table 2 . The average value for mean systolic stress $\left(360 \pm 37 \mathrm{~g} / \mathrm{cm}^{2}\right)$ was well within the range of normal in our laboratory $\left(286-434 \mathrm{~g} / \mathrm{cm}^{2}\right)$. This is the expected result in normotensive patients with a normal LV mass/volume ratio and a normal EF. ${ }^{19}$ These observations are in accordance with the published data indicating a normal afterload-shortening relationship in chronic compensated MR.

\section{DISCUSSION}

In chronic MR, the left ventricle functions as a double outlet chamber with retrograde flow passing through an orifice area that is only about $15 \%$ of that of a normal LV outflow area. ${ }^{21}$ This difference alone would be expected to minimise MRV. However, retrograde flow is influenced by other factors acting in concert with the orifice area, namely the magnitude and duration of the pressure gradient across the regurgitant orifice-all of which affect impedance and resistance. Thus, in MR the relative retrograde and forward flows depend on relative impedance and resistance of the two outlets. The model indicates that the impedance to retrograde flow exceeds that of forward flow until the regurgitant fraction exceeds $57 \%$. Only in the most severe MR (eg, acute severe MR) is the impedance to retrograde flow less than the impedance to forward flow. In the 10 patients (regurgitant fraction $=53 \pm 4 \%$ ), the average ratio of retrograde impedance to forward impedance was $1.22 \pm 0.19$, which is in close agreement with the model (figure 1). It appears, therefore, that it is misleading, if not incorrect, to describe the pathophysiology of chronic MR as a low impedance leak into the left atrium. It might be most appropriate to limit the low impedance' term to patients with the most severe MR (ie, a regurgitant fraction $>57 \%$ ).

\section{Afterload and impedance}

The LV wall stress data, in agreement with other published information, confirm a normal afterload in chronic compensated MR. The development of eccentric hypertrophy results in a compensatory phase of chronic MR, with normal preload (at the sarcomere level), normal afterload (at the ventricular level) as well as normal contractility and EF; the total SV is increased as a result of the large $\mathrm{EDV}^{3}$ Thus, the large total SV is "mediated through a normal performance of each unit of an enlarged circumference". 22 These mechanical considerations are not inconsistent with the hydraulic description of an enlarged double outlet ventricle with a low total impedance to emptying and a large total SV. Any nominal disparity between the concept of low total impedance and normal afterload is a consequence of the fact that impedance is a hydraulic term expressed in terms of pressure and volume within the chamber, while afterload is a mechanical term expressed as a force per unit cross-sectional area of the myocardium. Indeed, the low total hydraulic load seen in the double outlet ventricle has an impact on total SV, but such a change in impedance is associated with compensatory adjustments in systolic wall stress that more adequately predict changes in the EF. ${ }^{4}$ These concepts of total impedance and global afterload are complimentary and they operate in a synergistic fashion to provide a complete hydraulic and mechanical description of LV performance and function in chronic MR.

\section{Vasodilators}

With few exceptions, the use of vasodilators and agents that inhibit or block the renin-angiotensin-aldosterone system in asymptomatic patients with primary MR has not been proven to provide clinical benefit. ${ }^{23}$ This has been explained by the fact that an isolated change in the systolic pressure would have only a modest effect on MRV because of the square root factor in the orifice equation. ${ }^{24}$ Our current analysis suggests a second explanation. That is, if the regurgitant orifice area remains constant, a reduction in LV systolic pressure with a vasodilator would have a similar effect on retrograde and forward impedance, and little change in the regurgitant fraction. By contrast, changes in the MRV may be seen if vasodilator therapy or other haemodynamic interventions are associated with changes in the regurgitant orifice area. ${ }^{24} 25$

In this study, impedance spectra, as are calculated in studies of aortic input impedance, were not determined. Such methodology requires simultaneous measurement of pressure and volume transients during ejection, and conversion of the instantaneous measurements to Fourier series for analysis. Such measurements of pressure and flow in the regurgitant outlet could not be made and therefore it was not possible to generate left atrial input impedance spectra. Rather, it was necessary to use an index of impedance and a mathematical model. The resistance data support the conclusions based on the impedance index, but lacking true impedance spectra, cautious interpretation is necessary. Our conclusions will need to be confirmed with rigorous principles of haemodynamics and more patients with a wide spectrum of regurgitation severity. It should also be recognised that impedance, like wall stress, is not constant during ejection and that calculations of the time course of impedance could not be made. Mean flow rates during ejection were used to determine the impedance index. This could obscure instantaneous changes that might be detected by more precise methods. Finally, reliable measurements of LV volumes and other derived parameters are of signal importance in the evaluation of the severity of MR. ${ }^{26}$ The potential problems of volume measurement must be acknowledged. The model, however, is based on mathematical principles and is not based on volume-measurement methodology.

\section{CONCLUSION}

In chronic MR, the myocardial stress-shortening relations remain normal, while the double outlet of an enlarged ventricle

\section{Key messages}

What is already known on this subject?

The concept of a 'low impedance leak into the left atrium' in chronic mitral regurgitation is widely accepted, but there are no published impedance or resistance data that support this notion.

\section{What might this study add?}

In chronic mitral regurgitation, the enlarged double outlet ventricle exhibits a low total impedance to emptying with a large stroke volume, but the impedance to regurgitant flow is not lower than that to forward flow. These unique observations on hydraulic loads and blood flow complement studies indicating normal afterload-ejection fraction relations in chronic mitral regurgitation.

How might this impact on clinical practice? Clarification of the pathophysiology of mitral regurgitation and the use of quantitative metrics will enlighten management decisions. 
provides a mechanism for a low total impedance to ejection and a large total SV. However, the impedance to retrograde flow exceeds the impedance to forward flow in severe MR with a regurgitant fraction in the range of $50 \%-56 \%$, as well as in those with a regurgitant fraction that is $<50 \%$. Thus, it is misleading to describe the pathophysiology of MR as 'a low impedance leak into the left atrium'.

Contributors All the authors met the International Committee of Medical Journal Editors recommendations for authorship.

Competing interests None declared.

Ethics approval Institutional Review Board, Lahey Hospital and Medical Center.

Provenance and peer review Not commissioned; externally peer reviewed.

\section{REFERENCES}

1 Ross J Jr. Afterload mismatch in aortic and mitral valve disease: implications for surgical therapy. J Am Coll Cardiol 1985;5:811-26.

2 Bonow RO. Chronic mitral regurgitation and aortic regurgitation: have indications for surgery changed? J Am Coll Cardiol 2013;61:693-701.

3 Gaasch WH, Meyer TE. The left ventricular response to mitral regurgitation: implications for management. Circulation 2008:118:2298-303.

4 Pouleur H, Covell JW, Ross J Jr. Effects of alterations in aortic input impedance on the force-velocity-length relationship in the intact canine heart. Circ Res 1979;45:126-35

5 Nichols WW, Pepine CJ. Left Ventricular afterload and aortic input impedance: implications of pulsatile blood flow. Prog Cardiovasc Dis 1982;24:293-306.

6 Briand M, Dumesnil JG, Kadem L, et al. Reduced systemic arteria compliance impacts significantly on left ventricular afterload and function in aortic stenosis: implications for diagnosis and treatment. J Am Coll Cardiol 2005;46:291-8.

7 Shah SP, Kumar A, Draper TS, et al. Hypertension in patients with severe aortic stenosis: emphasis on antihypertensive treatment and the risk of syncope. Curr Hyperten Rev 2014;10:149-54.

8 Kelly RP, Ting C, Yang TM, et al. Effective arterial elastance as index of arterial vascular load in humans. Circulation 1992;86;513-21.

9 Harris W. Systolic time intervals in the noninvasive assessment of left ventricular performance in man. In Mirsky I, Ghista DN, Sandler H, eds. Cardiac mechanics. New York: John Wiley and Sons Inc, 1994:233-92.
10 Zoghbi WA, Enriquez-Sarano M, Foster E, et al. Recommendations for evaluation of the severity of native valvular regurgitation with two-dimensional and Doppler echocardiography. J Am Soc Echocardiography 2003;16:777-802.

11 Mehrotra P, Labib SB, Schick EC. Differential effects of dobutamine versus treadmill exercise on left ventricular volume and wall stress. J Am Soc Echocardiography 2012;25:911-18.

12 Draper TS, Silver JS, Gaasch WH. Adverse structural remodeling of the left ventricle and ventricular arrhythmias in patients with depressed ejection fraction. J Card Fail 2015;21:97-102

13 Teichholz LE, Kreulen T, Herman MV, et al. Problems in echocardiographic volume determinations: echocardiographic-angiographic correlations in the presence or absence of asynergy. Am J Cardiol 1976;37:7-11.

14 Dumesnil JG, Dion D, Yvorchuk K, et al. A new, simple, and accurate method for determining ejection fraction by Doppler echocardiography. Can J Cardiol 1995:11:1007-14.

15 Devereux RB, Alonso DR, Lutas EM, et al. Echocardiographic assessment of left ventricular hypertrophy: comparison to necropsy findings. Am J Cardiol 1986;57:450-8

16 Gaasch WH, Zile MR. Left ventricular structural remodeling in health and disease: with special emphasis on volume, mass, and geometry. J Am Coll Cardiol 2011;58:1733-40.

17 Beiser A. Applied physics (4 ${ }^{\text {th }}$ edition), Schaum's outline series. New York: McGraw Hill, 2004:290-1

18 Ford LE, Feldman T, Chiu C, et al. Hemodynamic resistance as a measure of functional impairment in aortic valvular stenosis. Circ Res 1990;66:1-7.

19 Arts T, Bovendeerd PHM, Prinzen FW, et al. Relation between left ventricular cavity pressure and volume, and systolic fiber stress and strain in the wall. Biophys $J$ 1991;59:93-102.

20 Gaasch WH, Battle WE, Oboler AO, et al. Left ventricular stress and compliance in man: with special reference to normalized ventricular function curves. Circulation 1972:45:746-62

21 Gorlin R, Dexter L. Hydraulic formula for the calculation of the cross sectional area of the mitral valve during regurgitation. Am Heart J 1952;43:188-205.

22 Ross J Jr. Adaptations of the left ventricle to chronic volume overload. Circ Res 1974;35(Suppl II):64-70.

23 Borer JS, Sharma A. Drug therapy for heart valve diseases. Circulation 2015:132:1038-45.

24 Levine HJ, Gaasch WH. Vasoactive drugs in chronic regurgitant lesions of the mitral and aortic valves. J Am Coll Cardiol 1996;28:1083-91.

25 Chattterjee K, Parmley WW, Swan HJC, et al. Beneficial effects of vasodilator agents in severe mitral regurgitation due to dysfunction of subvalvular apparatus. Circulation 1973;48:684-90.

26 Grayburn PA, Carabello B, Hung J, et al. Defining "severe" secondary mitral regurgitation. J Am Coll Cardiol 2014;64:2792-801. 